\title{
Pour une génétique des cycles romanesques
}

\section{Alain Pagès}

\section{OpenEdition}

\section{Journals}

Édition électronique

URL : https://journals.openedition.org/genesis/1265

DOI : 10.4000/genesis. 1265

ISSN : 2268-1590

\section{Éditeur :}

Presses universitaires de Paris Sorbonne (PUPS), Société internationale de génétique artistique littéraire et scientifique (SIGALES)

\section{Édition imprimée}

Date de publication : 9 mai 2016

Pagination : 7-16

ISBN : 9791023105315

ISSN : 1167-5101

\section{Référence électronique}

Alain Pagès, «Pour une génétique des cycles romanesques », Genesis [En ligne], 42 | 2016, mis en ligne le 03 juin 2016, consulté le 17 janvier 2023. URL : http://journals.openedition.org/genesis/1265 ; DOI : https://doi.org/10.4000/genesis.1265 


\section{Pour une génétique des cycles romanesques}

e numéro se propose d'ouvrir, dans le champ conceptuel de la génétique, un nouveau chantier, celui d'une réflexion sur les cycles romanesques. Au-delà d'une génétique des æeuvres individuelles (étudiées à travers leurs variantes scripturales, leurs ébauches ou leurs scénarios), il souhaite définir les principes d'une génétique des æuvres concaténées, liées par la structure du cycle. Pour reprendre une image employée par Olivier Lumbroso dans l'article qui prolonge cette introduction, il s'agit de changer d'échelle, d'ouvrir un nouvel espace à la perception des phénomènes de genèse. Les exemples rassemblés ici s'efforcent de dessiner un vaste panorama historique, qui va de La Comédie humaine de Balzac aux Thibault de Roger Martin du Gard et aux Hommes de bonne volonté de Jules Romains.

"Ce qui me fascine, ce sont les romans-mondes, tramés d'histoire », s'exclame Hédi Kaddour dans l'entretien que nous publions plus loin. En commentant le travail qui a étéle sien quand il s'est lancé dans l'écriture de Waltenberg, Hédi Kaddour reprend à son compte l'expression de "roman-monde» forgée par Tiphaine Samoyault - proche d'autres formulations, avancées dans la même perspective, celles d' «œuvre-monde» ou de «livre-monde ${ }^{1}$ ». De tels romans, en liant entre eux récits et personnages, ont l'ambition de représenter la totalité d'une réalité historique et sociale. Mais si on les considère du point de vue de l'énonciation, on pourrait parler aussi, à leur propos, d' «euvres-vies», car leur écriture engage, en règle générale, une vie entière. Un rêve esthétique préside à leur création, celui d'un texte qui serait nourri par la matière de toute une existence, dont l'écriture n'aurait jamais de terme, et qui trouverait dans cette durée même la possibilité de multiples reprises justifiant sa raison d'être. L'œuvre-vie est indissociable de l'œuvre-monde. L'engagement qui se trouve à l'origine de la première autorise l'ambition dont se réclame la seconde. Cet idéal est illustré par le modèle fondateur que représentent les Essais de Montaigne. Aussi n'est-il pas étonnant que la figure de Montaigne revienne à deux reprises dans les pages qui suivent: Olivier Lumbroso lui accorde une grande importance, en rappelant que l'auteur des Essais a constitué l'une des premières lectures de Zola; et Aude Leblond montre le rôle qu'elle a joué dans la réflexion de Roger Martin du Gard.

\footnotetext{
1. Voir Tiphaine Samoyault, Romans-mondes : formes de la totalisation romanesque au XXe siècle, thèse de l'université de Paris VIII, Lille, ANRT, 1996; «La reprise (note sur l'idée de roman-monde)», Romantisme, $\mathrm{n}^{\circ} 136$, «L'œuvre-monde au XIXe siècle», 2007, p. 95-104; Dominique Massonnaud, Faire vrai. Balzac et l'invention de l'œuvre-monde, Genève, Droz, 2014. Sur la notion de «livre-monde», voir Aude Leblond, Sur un monde en ruines. Esthétique du roman-fleuve, Paris, Honoré Champion, 2015, p. 13 et 240-241.
} 


\section{Questions de terminologie}

Quel terme employer? «Cycle» ou « série»? Pour Balzac et Zola, la question ne se pose pas. Ils ne parlent pas de «cycle»: Balzac ignore ce mot lorsqu'il évoque son œuvre, tandis que Zola emploie celui de «série». Car le terme de «cycle» n'appartient pas au métalangage de la critique littéraire, à l'époque où ils écrivent. Comme le rappelle ici Christophe Pradeau, il surgira plus tard, dans le courant des années 1890 et au début du XXe siècle, à la suite de la réflexion conduite par Albert Thibaudet sur l'évolution du roman.

De Balzac à Jules Romains les formes évoluent. C'est Romain Rolland, on le sait, qui invente l'expression de "roman-fleuve», bientôt consacrée par les travaux de Thibaudet. Faut-il alors opposer les deux concepts, «cycle» d'un côté, «roman-fleuve » de l'autre, pour souligner le changement de perspective qui s'est opéré à partir de Romain Rolland? Revenant sur cette discussion théorique, Christophe Pradeau propose d'adopter une expression de caractère synthétique qui offre l'avantage d'une vision unifiée, conforme, d'ailleurs, à la problématique d'ensemble sur laquelle repose ce numéro. En le suivant, on distinguera donc les cycles à "entrées multiples», fondés sur des récits qui demeurent autonomes (La Comédie humaine, Les Rougon-Macquart), et les cycles à «entrée unique » qui postulent une continuité de lecture (Jean-Christophe, À la recherche du temps perdu, Les Thibault, Les Hommes de bonne volonté, c'est-à-dire les romans-fleuves de la première moitié du XXe siècle).

Dès que l'on regarde les choses d'un peu plus près, cependant, on s'aperçoit que cette opposition entre "entrée unique » et "entrées multiples» n'est pas aussi simple qu'elle le paraît, au premier abord, et que la réalité est plus complexe. Les cycles à entrées multiples peuvent offrir, en dépit de leur fragmentation, des enchaînements narratifs : la trilogie de l'Histoire des Treize qu'étudie l'article de Thomas Conrad (Ferragus, La Duchesse de Langeais, La Fille aux yeux d'or) constitue un microcycle autonome au sein de La Comédie humaine; dans Les Rougon-Macquart, les romans du cycle de Plassans (La Fortune des Rougon, La Conquête de Plassans, La Faute de l'abbé Mouret et Le Docteur Pascal) possèdent un statut comparable; ils reposent sur une unité de lieu (la ville de Plassans) et une unité d'action (l'histoire familiale) qui favorisent une lecture en continu. Inversement, les cycles à entrée unique peuvent comporter des îlots narratifs indépendants de l'ensemble. Christophe Pradeau et Sophie Guermès le soulignent à propos de Proust et de Jules Romains : que l'on songe, dans la Recherche, au statut privilégié que possède l'histoire de Swann, ou, dans le cas de Jules Romains, à ces arrêts, ces pauses dans un espace particulier qui rompent la chronologie des Hommes de bonne volonté en offrant au lecteur des moments de respiration lui permettant de se libérer des contraintes imposées par la suite romanesque.

\section{Quel dossier de genèse?}

Sur quel type de dossier de genèse une génétique des cycles romanesques peut-elle s'appuyer? Alors que la génétique des textes privilégie le regard sur l'œuvre en train de surgir, elle s'intéresse à la série, à la durée chronologique dans laquelle les textes s'inscrivent. L'examen des exemples dont traite ce numéro montre que deux sortes d'éléments peuvent entrer dans ce dossier : des notes préparatoires, définissant les conditions d'un 
programme narratif au long cours; et un paratexte, composé de préfaces ou de schémas, expliquant, à l'attention des lecteurs, les raisons du projet qui est mis en avant. Les notes préparatoires laissées par l'écrivain fournissent la base du dossier de genèse 2 . Mais, si l'on s'intéresse au projet du cycle dans son ensemble, elles apparaissent souvent lacunaires. L'exemple offert par Zola - un manuscrit liminaire contenant d'importantes «notes préparatoires » qui lancent un vaste programme narratif - possède quelque chose d'exceptionnel. Dans la plupart des cas, il faut se contenter de notes générales assez succinctes : Sophie Guermès, par exemple, insiste sur la concision des plans et des fiches-personnages laissés par Jules Romains. Pour combler ce manque, la recherche génétique doit donc se tourner vers le paratexte, en lui accordant toute la place qu'il mérite.

En raison de l'importance qu'il possède pour la découverte de l'œuvre, celui-ci ne peut être contourné : la préface de La Fortune des Rougon, au début des Rougon-Macquart, ou la préface des Hommes de bonne volonté constituent des seuils obligatoires que l'on est forcé de franchir, si l'on veut comprendre ce que l'écrivain propose. Les préfaces possèdent deux fonctions : annonciatrices, elles posent le cadre du récit qui va suivre, mais sans tout dévoiler, cependant, ce qui serait, d'ailleurs, impossible (Jules Romains, par exemple, se refuse à dire de combien d'épisodes doit se composer le cycle des Hommes de bonne volonté); régulatrices, elles font le point sur l'avancée du projet, en invitant le lecteur à tenir bon, jusqu'à la fin de l'entreprise.

Un schéma tel que l'arbre généalogique dessiné par Zola attire l'attention : on le retient à cause de son caractère inhabituel; il possède une vertu publicitaire qui confère à l'auvre une aura particulière associant les prestiges de la science aux mystères de l'hérédité. Pour Les Rougon-Macquart, il devient une sorte de symbole qui résume tout le cycle : un manuel scolaire évoquant aujourd'hui l'œuvre de Zola, un site Internet consacré à l'écrivain ne manquent pas de le reproduire; l'oublier apparaîtrait aux auteurs de ces exposés de vulgarisation comme une grave lacune ${ }^{3}$ (fig. 1).

La disposition des titres (construits sur deux niveaux, jouant sur le global et le particulier), la quatrième de couverture ou le prière d'insérer favorisent la lisibilité de la série, en soulignant sa structure. Ils fournissent des repères précieux pour saisir la genèse du cycle. Des informations complémentaires peuvent entrer en jeu. Ainsi les volumes de la «Bibliothèque Charpentier» qui publient Les Rougon-Macquart, au XIXe siècle, donnent, à gauche de la page de titre, la liste des volumes parus avec, pour chacun d'entre eux, l'indication du nombre d'éditions qui a été atteint (fig. 2). Ces données quantitatives permettent d'établir un classement. Elles montrent l'unité de la série, tout en suggérant que chaque volume

\footnotetext{
2. On ne soulignera jamais assez l'importance des legs adressés à la postérité et que les bibliothèques ont pour fonction de conserver : dossiers de Zola et de Jules Romains déposés à la Bibliothèque nationale; dans le cas de Roger Martin du Gard, cette «malle de documents», avec sa «boîte noire», qu'évoque Aude Leblond à propos du dernier projet romanesque de l'écrivain, Le Lieutenant-Colonel de Maumort. C'est un point sur lequel les théoriciens de la critique génétique ont, très tôt, mis l'accent : voir, par exemple, Almuth Grésillon, Éléments de critique génétique, Paris, PUF, 1994, p. 12, 77-78; réédition : CNRS Éditions, 2016.

3. De cette célébrité universelle on trouve un écho dans le roman de Fabrice Humbert, Éden Utopie, publié au début de l'année 2015, qui, reprenant le projet zolien - raconter l'histoire d'une famille double, divisée en Rougon et en Macquart -, s'ouvre, en signe de reconnaissance intertextuelle, sur un arbre familial.
} 


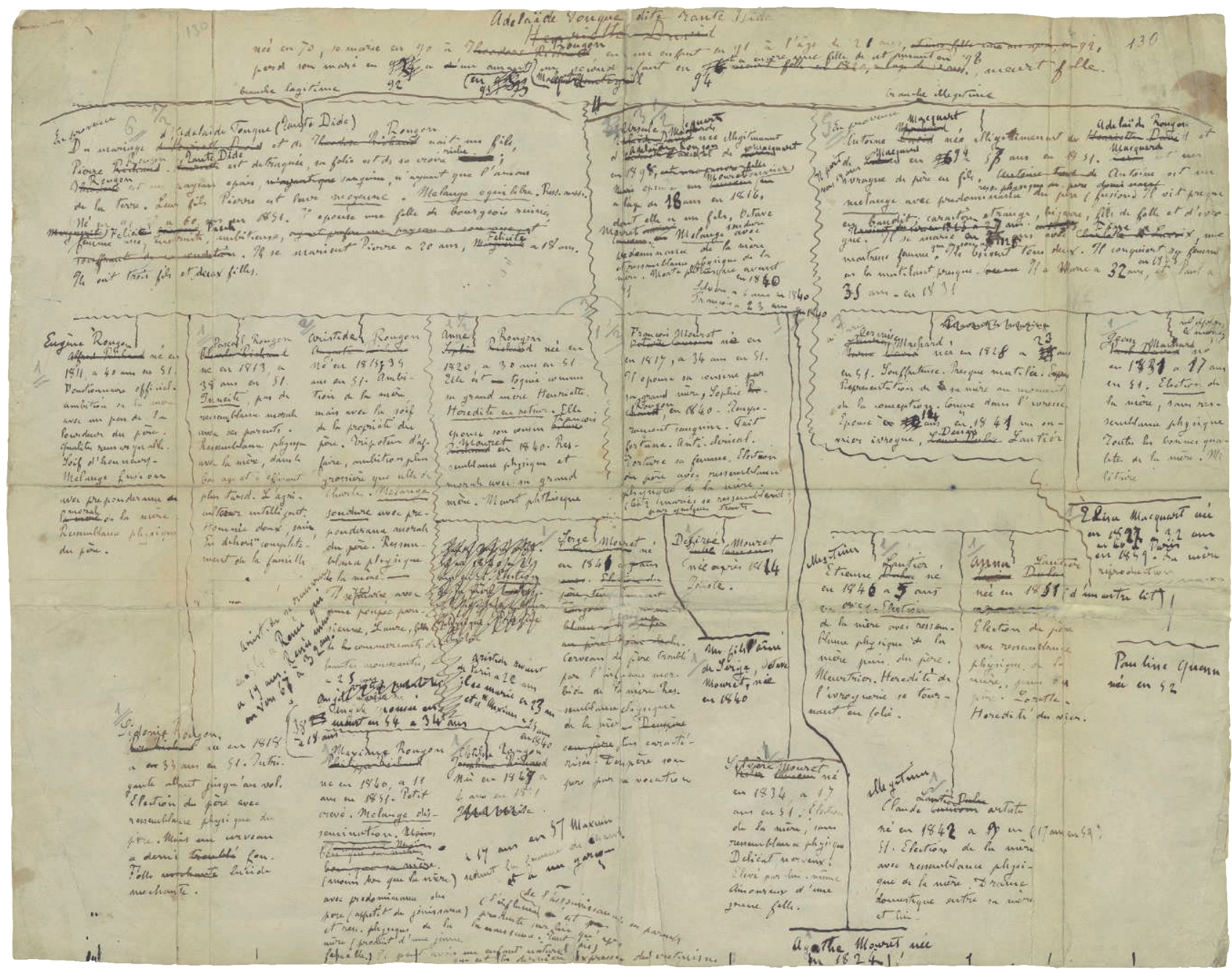

Fig. 1 : Émile Zola, Premier arbre généalogique des Rougon-Macquart (1868); voir aussi p. 40 et p. 99 (BnF, NAF 10345, f 267)

poursuit son existence propre, porté par ses éditions successives. Sur ce paratexte liminaire, une sorte de course imaginaire rassemble tous les romans du cycle. On les imagine dans le groupe compact qu'ils forment, rivalisant d'efforts entre eux, tendus vers le poteau d'arrivée que dressera le dernier épisode. En 1878, par exemple, lorsque paraît Une page d'amour, L'Assommoir arrive en première position, suivi par La Curée, puis par quatre volumes placés ex aequo, La Fortune des Rougon, Le Ventre de Paris, La Faute de l'abbé Mouret et Son Excellence Eugène Rougon, tandis que La Conquête de Plassans termine en queue du peloton. En 1894, au moment où la série vient de s'achever, avec la publication du Docteur Pascal, La Débâcle caracole en tête (182000 exemplaires), suivi par Nana (171 000 exemplaires), L'Assommoir (132 000 exemplaires), La Terre (107 000 exemplaires) et Germinal (94 000 exemplaires); classés derniers, La Fortune des Rougon et La Conquête de Plassans font figure de cancres, avec seulement 29000 exemplaires.

Dans une génétique des cycles romanesques, les préfaces, la correspondance ou les confidences de l'écrivain recueillies par le biais d'interviews jouent un rôle plus important que celui qu'elles possèdent dans une génétique des textes. De même, la dimension biogra- 


\section{G. CHARPENTIER et E. FASQUELLE, Éditeurs 11, RUE DE GRENELLE, 11}

\section{OUVRAGES DU MÊME AUTEUR \\ DANS LA BIBLIOTHÈQUE-GHARPENTIER \\ à 3 fr. 50 chaque volume.}

\section{LES ROUGON-MACQUART}

HISTOIRE NATURELLE ET SOCIALE D'UNE FAMIILE SOUS LE SEGOND EMPIRE LA FORTUNE DES ROUGON. ......... $29^{\circ}$ mille. 1 vol. LA CUREE. ................. 40 mille. 1 vol. LE VENTRE DE PARIS. ............. 36, mille. 1 vol. LA CONQUÊTE DE PLASSANS. ........ $29^{\circ}$ mille. 1 vol. LA FAUTE DE L'ABBE MOURET. ...... $44^{\circ}$ mille. 1 vol. SON EXCELLENCE EUGÉNE ROUGON. ..... $28^{\circ}$ mille. 1 vol. L'ASSOMMOIR. ............. 132 mille. 1 vol. UNE PAGE D'AMOUR. ............. 80. mille. 1 vol. NANA. ................ 171 mille. 1 vol. POT-BOUILLE................ $85^{\prime}$ mille. 1 vol. AU BONHEUR DES DAMES. ...........620 mille. 1 vol. LA JOIE DE VIVRE. ............. $48^{\circ}$ mille. 1 vol. GERMINAL. ............... 940 mille. 1 vol. L'CEUVRE. . . . . . . . . . . 55 mille. 1 vol. LA TERRE. ............... 107 mille. 1 vol. LE REVE. . . . . . . . . . . . $94^{\circ}$ mille. 1 vol. LA BÊTE hUMAINE. ........... $88^{\circ}$ mille. 1 vol. L'ARGENT. .............. $83^{\circ}$ mille. 1 vol. LA DEBACLE. .............. $182^{\circ}$ mille. 1 vol. LE DOCTEUR PASCAL............ 88 . mille. 1 vol.

Fig. 2 : Les Rougon-Macquart dans la Bibliothèque Charpentier en 1894

phique y est plus fortement présente. C'est ce que montre Céline Grenaud-Tostain lorsqu'elle compare, en entrant dans le cour même de l'expérience créatrice, Zola et Romain Rolland. Au démarrage de leur écriture cyclique, les deux écrivains choisissent d'affronter différemment la question de leur implication personnelle. Par une sorte d'ascétisme du nulla dies sine linea symbolisé par la solitude que lui offre sa tour de Médan, Zola choisit un modèle du dépassement, dans lequel les événements de l'existence sont volontairement coupés du processus de l'écriture. Romain Rolland, au contraire, en imaginant un personnage central capable de relayer ses propres préoccupations intellectuelles, peut introduire des liens plus importants entre la dynamique de son écriture et ce que la vie quotidienne lui apporte, dans le flux de ses émotions et de ses suggestions.

Faut-il aller plus loin encore, et chercher dans la fiction elle-même des éléments susceptibles de nourrir le dossier de genèse? C'est la voie dans laquelle s'engagent les articles de Thomas Conrad et de Christophe Pradeau.

D'après Thomas Conrad, La Comédie humaine trouve son origine dans un travail interne du texte sur lui-même. Elle repose sur un «protocycle» : la trilogie de l'Histoire des Treize. Dans ce récit fondateur le principe du retour des personnages se met en place; la vision sociologique s'approfondit, dépassant le modèle antique du héros aristocratique pour embrasser toute la complexité du réel. Ainsi, par sa dynamique propre, l'écriture libère-t-elle 
les forces de l'imaginaire, amenant le romancier à dépasser les influences héritées de la tradition pour le conduire à l'intuition créatrice du concept unificateur de "Comédie humaine».

Christophe Pradeau, de son côté, avance la notion de "fable génétique ». Située dans le discours critique, issue des témoignages dont se compose la biographie de l'écrivain, ou placée au cour même de la fiction, celle-ci utilise un registre métaphorique ou bien allégorique pour décrire l'imaginaire du cycle. Zola, songeant à l'exemple de Balzac, voit La Comédie humaine comme une "tour de Babel que la main de l'architecte n'a pas eu et n'aurait jamais eu le temps de terminer», comme "un entassement de palais et de bouges, un de ces monuments cyclopéens comme on en rêve, pleins de salles splendides et de réduits honteux, coupé par de larges promenoirs et par des corridors étroits où l'on ne passe qu'en rampant ${ }^{\Perp}$. De même, Jules Romains représente l'espace urbain des Hommes de bonne volonté à travers le "Conte des 365 appartements» qui évoque l'image d'un Paris mystérieux, aux multiples réseaux, où l'on circule d'une manière souterraine, en passant clandestinement d'un lieu à un autre.

Olivier Lumbroso adopte une perspective similaire lorsqu'il souligne les effets de mise en abyme inscrits dans la série des Rougon-Macquart. Placée aux deux extrémités du cycle, dans La Fortune des Rougon et dans Le Docteur Pascal, la «vision prophétique puis rétrospective de Pascal-Zola» est complétée dans L'Cuvre, au centre du cycle, par la "vision artistique de l'écrivain Sandoz», qui livre l'argument de la série : "Je vais prendre une famille, et j'en étudierai les membres, un à un, d'où ils viennent, où ils vont, comment ils réagissent les uns sur les autres; enfin, une humanité en petit, la façon dont l'humanité pousse et se comporte 5 ...»

Par ces fables les romanciers du cycle ouvrent à leurs lecteurs les portes du laboratoire dans lequel ils travaillent. Comment ne pas transformer en destinée romanesque ce rêve de gloire et cette hantise de l'échec qui les torturent tous? En réunissant les personnages de Claude et de Sandoz, qui apportent des réponses différentes aux problèmes posés à l'artiste par les impératifs de la création artistique, L'Euvre a inscrit au cour de son intrigue cette quête qui est celle de la continuité dans l'écriture.

\section{Portrait du romancier « cycliste $^{6} »$}

Pour un écrivain, la décision qui consiste à s'engager non dans une æuvre unique, mais dans une suite d'œuvres, dont la composition s'étalera sur plusieurs années, représente un choix lourd de conséquences. Ce moment constitue un tournant dans une existence. Il s'ac-

\footnotetext{
4. Les Romanciers naturalistes, Euvres complètes, Paris, Nouveau Monde Éditions, t. X, 2004, p. 445. En 1894, dans un entretien avec Charles Morice portant sur le symbolisme, Zola reprendra à son compte cette image des «corridors étroits»: «Mes livres sont des labyrinthes où vous trouveriez, en y regardant de près, des vestibules et des sanctuaires, des lieux ouverts et des lieux secrets, des corridors sombres, des salles éclairées » (Entretiens avec Zola, éd. Dorothy Speirs et Dolorès Signori, Ottawa, Les Presses de l'Université d'Ottawa, 1990, p. 140).

5. L'Euvre, Paris, Gallimard, coll. «Bibliothèque de la Pléiade», t. IV, 1967, p. 162.

6. L'expression se trouve sous la plume d'Albert Thibaudet qui, non sans humour, compare la situation des auteurs de cycles romanesques à celle des coureurs du Tour de France (Histoire de la littérature française, Paris, Stock, 1936, p. 547).
} 
compagne de sentiments contradictoires, comme le montrent les exemples analysés dans ce numéro. Au bonheur lié à l'intuition créatrice qui s'est emparée de l'esprit s'oppose la crainte d'un échec possible, l'angoisse devant la réalité d'une tâche qui semble soudain insurmontable, mais que l'on a, pourtant, volontairement programmée.

Le bonheur, c'est-comme l'évoque Christophe Pradeau-celui de Balzac se précipitant chez sa sœur, Laure Surville, et lui criant : «Saluez-moi... car je suis tout bonnement en train de devenir un génie! », à la suite de cette illumination rétrospective qui vient de le conduire à la vision systématique de son æeuvre. C'est aussi celui de Zola exposant fièrement à son éditeur, Albert Lacroix, l'originalité d'un projet qui fera pendant à l'illustre modèle de La Comédie humaine; et lui déclarant qu'il entend faire "pour le Second Empire ce que Balzac a fait pour le règne de Louis-Philippe », mais en se montrant "plus méthodique » que son prédécesseur ${ }^{7}$. C'est encore Jules Romains, ébloui par la découverte de l'unanimisme qui va lui fournir le cadre philosophique à l'œuvre à venir. On notera que cette illumination créatrice, qui possède une dimension mystique (comparable à une révélation d'essence religieuse), se produit au terme d'un cheminement initiatique. Elle surgit après la publication d'œuvres réflexives (essais ou fictions proches de l'essai) qui peuvent être considérées comme des prologues théoriques : pour Proust, le Contre Sainte-Beuve et Jean Santeuil avant la Recherche; pour Roger Martin du Gard, Jean Barois avant Les Thibault; pour Jules Romains, La Vie unanime avant Les Hommes de bonne volonté.

À la joie créatrice s'oppose le sentiment inverse d'une peur devant l'inachèvement. Ce sentiment éclate dans la préface aux Hommes de bonne volonté - un document étonnant dont Sophie Guermès souligne la richesse. Jules Romains manifeste sa joie dans les premières lignes. Mais très vite, en s'adressant à son lecteur, il lui fait part de ses craintes. Et toute la préface des Hommes de bonne volonté se transforme bientôt en une vaste captatio benevolentiae, un plaidoyer tourné vers le lecteur auquel l'écrivain demande d'être patient et de ne pas être effrayé par l'édifice dont les pierres vont être progressivement assemblées.

Même angoisse chez Rosny, comme le montre Jean-Michel Pottier, en analysant une situation pourtant bien différente. Rosny est obsédé par l'idée d'introduire dans son ouvre une cohérence de nature cyclique. S'adressant à ses lecteurs, il s'efforce de montrer, dans ses préfaces, la continuité de ses schémas cycliques. Prisonnier des modèles que lui livrent ses prédécesseurs, on le voit hésiter entre la vision balzacienne du cycle reconnu a posteriori et la méthode zolienne du cycle défini a priori. Avec son frère il s'engage dans la pratique de l'écriture collaborative pour l'abandonner, au bout de quelques années (elle aurait pu se révéler féconde, pourtant, quand on songe aux duos que formeront, plus tard, certains auteurs de romans policiers ou de romans d'espionnage ${ }^{8}$ ). Jouant sur plusieurs types de regroupements, il s'est efforcé d'unifier son ouvre à travers trois formules cycliques : le «cycle des mondes sociaux» (auquel appartient, par exemple, Le Bilatéral, en 1887), le "cycle des mondes légendaires » (illustré par son roman le plus célèbre, La Guerre du feu, en 1911) et les « romans du monde des variants » (comme La Mort de la terre, en 1912). Cas passionnant

7. «Premier plan remis à Lacroix», dans La Fabrique des Rougon-Macquart, éd. C. Becker, Paris, Honoré Champion, 2003, t. I, p. 330.

8. Voir, à ce sujet, l'ouvrage de Michel Lafon et Benoît Peeters, Nous est un autre. Enquête sur les duos d'écrivains, Paris, Flammarion, 2006. 
que celui de Rosny... Il témoigne d'une construction cyclique en mouvement, dont la genèse est issue d'une relation dialectique qui s'est établie entre les intentions de l'auteur et les réactions des lecteurs.

Un tel sentiment d'angoisse surgit chez des écrivains qui ont le sentiment d'arriver trop tard, d'entrer dans le champ littéraire après des aînés qui les écrasent, et contre l'ombre desquels ils devront lutter : telle est la situation de Rosny face à Zola, de Jules Romains ou de Roger Martin du Gard face à leurs grands modèles du siècle précédent. L'écrivain "cycliste » est, avant tout, un héritier, comme l'a remarqué Albert Thibaudet (que citent, sur ce point, Olivier Lumbroso et Christophe Pradeau). Il reprend, il recommence, mais en étant contraint de faire autre chose. Devant la fatalité de cet impossible recommencement, une sorte de vertige s'empare de lui, surtout quand la tragédie de l'Histoire s'en mêle, en brouillant les repères. Ainsi en est-il de Roger Martin du Gard, comme le montre Aude Leblond: le traumatisme de la Première Guerre mondiale remet en cause son projet, d'une manière soudaine, en brisant toute relation avec l'époque antérieure qu'il souhaitait représenter.

L'écriture cyclique finit par dévorer l'existence tout entière. On connaît la situation tragique de Proust demandant à la destinée de lui accorder suffisamment de forces pour qu'il ait le temps d'achever son œuvre. Zola a subi la même emprise. Rien ne le laissait présager, pourtant, lorsque, le 12 juillet 1872, il adresse au directeur de la Bibliothèque nationale cette simple demande : "Je vous prie de bien vouloir mettre à ma disposition une carte d'entrée à la salle de travail de la Bibliothèque. J'ai besoin de cette carte pour différents travaux littéraires 9 . " Quelques «travaux littéraires»? Ces travaux ne s'arrêteront jamais... Après Les Rougon-Macquart, Zola s'est engagé dans Les Trois Villes et Les Quatre Évangiles, en dépit de la lassitude qu'il ressentait ${ }^{10}$. Et quand il disparut (sans avoir pu écrire le quatrième tome de ses Évangiles), il songeait à un nouveau cycle encore, dont il parla à Maurice Le Blond, en juin 1902 : il rêvait, lui disait-il, à des drames qui seraient regroupés sous le titre de «La France en marche», car il voudrait faire pour la Troisième République ce qu'il avait fait pour le Second Empire, "une série d'œuvres, où se retrouver[ait] l'histoire naturelle et sociale de l'époque 11 ». Les Ébauches inédites dont Jean-Sébastien Macke assure la présentation dans ce numéro montrent que cette loi de l'écriture cyclique s'est appliquée également à des projets d'œuvres théâtrales conçus à la fin des années 1880 et au début des années 1890, entre 1887 et 1893.

$$
\text { *** }
$$

9. Lettre inédite, publiée par Agnès Sandras et Charles-Éloi Vial dans la chronique qui figure à la fin de notre dossier. 10. La notion de «protocycle», que nous avons évoquée plus haut, pourrait être appliquée à la genèse des RougonMacquart. Le cycle succède à des romans de jeunesse (La Confession de Claude, Thérèse Raquin et Madeleine Férat) qui forment une sorte de trilogie de la «femme maudite», comme l'a noté Henri Mitterand («Le troisième Zola», Il terzo Zola. Émile Zola dopo $i$ «Rougon-Macquart», éd. G.-C. Menichelli, Napoli, Istituto Universitario Orientale, 1990, p. 3). Celle-ci apparaît comme le protocycle de la série, le lieu où Zola s'exerce, abandonne le lyrisme romantique de La Confession de Claude pour découvrir le roman physiologique, tout en construisant une vision des rapports amoureux qui demeurera une constante dans toute son œuvre. Les Rougon-Macquart surgissent ainsi au confluent de deux lignes narratives : le protocycle de la femme maudite; et l'intertexte des mystères urbains, sur le modèle d'Eugène Sue, dont Zola a fait l'expérience en écrivant Les Mystères de Marseille en 1867.

11. M. Le Blond, Les Projets littéraires d'Émile Zola au moment de sa mort d'après des documents et des manuscrits inédits, Paris, Mercure de France, 1927, p. 21. 
Au terme de cette recherche, les questions que peut se poser une génétique des cycles romanesques apparaissent d'une façon assez claire.

Cette génétique doit d'abord s'interroger sur ce désir du cycle qui se trouve à l'origine de telles entreprises. Pourquoi vouloir engager tant d'efforts, sur de si nombreuses années? Il s'agit d'ajouter à l'œuvre une "beauté» que l'on pense décisive, comme l'indique ici Christophe Pradeau en citant l'exemple de Proust. L'enjeu concerne la réception : retenir l'attention des critiques en les mettant devant une construction monumentale (c'est-à-dire, au sens étymologique, digne de mémoire); et proposer aux lecteurs un enchaînement narratif qui puise aux sources du roman-feuilleton né avec la presse moderne. À la suite de Balzac et de Zola ce désir cyclique parcourra toute la fin du XIXe siècle 12 ; il s'épanouira dans les séries du roman populaire pour continuer à irriguer le roman, jusqu'au milieu du XXe siècle. S'il s'est essoufflé par la suite, il trouve, à notre époque, un étonnant relais dans les multiples séries télévisées qui, par leur capacité à représenter le réel, apparaissent comme les grandes héritières du réalisme balzacien - de House of Cards à Mad Men, Breaking Bad ou Borgen, pour ne citer que quelques titres récents. Nous n'en avons pas fini avec les cycles narratifs; ils appartiennent à notre modernité.

Cette génétique doit prendre en compte, également, la multiplicité des pratiques littéraires: cycles courts ou cycles longs; entrée unique ou entrées multiples; ceuvres entières soumises à la loi de l'écriture cyclique ou essais limités, accomplis à titre expérimental. Elle doit s'appuyer sur une enquête historique aussi large que possible. Les articles publiés dans ce numéro s'intéressent aux cycles les plus connus, de Balzac à Jules Romains, mais de nombreux autres exemples pourraient être cités, que l'on songe à des auteurs célèbres (Barrès, Aragon ou Sartre), à des écrivains qui bénéficient encore d'une notoriété importante (Georges Duhamel ou Jacques Chardonne), ou à des écrivains en partie tombés dans l'oubli (Joséphin Péladan, Victor et Paul Margueritte, Dubut de Laforest, dans la seconde moitié du XIXe siècle; René Béhaine, Abel Hermant, Robert Francis, Jacques de Lacretelle, au début du $\mathrm{XX}^{\mathrm{e}}$ siècle $\left.{ }^{13}\right)$.

Dernière remarque enfin, la génétique des cycles romanesques doit nécessairement repenser, pour ce qui la concerne, le statut de l'avant-texte. Elle se trouve dans une situation comparable à celle de la génétique des auvres théâtrales telle qu'elle a été analysée par Almuth Grésillon ${ }^{14}$. Un cycle romanesque regroupe des textes pour les faire jouer ensemble sur un vaste territoire imaginaire. C'est une sorte de mise en scène, comparable au travail

12. Si l'on prend l'exemple de la littérature naturaliste, on voit que cet impératif moral s'impose aux débutants, en aggravant la pression qu'exerce sur eux le poids des maitres : Léon Hennique donne à ses deux premiers romans (La Dévouée et L'Accident de Monsieur Hébert) le titre générique des «Héros modernes », annonçant ainsi une entreprise cyclique qu'il ne poursuivra pas; quelque temps avant sa mort, Robert Caze avait imaginé le projet d'un «Essai sur la société contemporaine» qui devait se composer de quatre séries, «les Filles, les Femmes, les Hommes, les Enfants » (voir R.-P. Colin, Dictionnaire du naturalisme, Tusson, Du Lérot, 2012, p. 105).

13. Citons les titres : les trilogies de Barrès, Le Culte du moi, Le Roman de l'énergie nationale et Les Bastions de l'Est; Les Chemins de la liberté de Sartre, Le Monde réel d'Aragon; la Chronique des Pasquier et Vie et aventure de Salavin de Georges Duhamel, les Destinées sentimentales de Jacques Chardonne; 1' «éthopée» de Joséphin Péladan, La Décadence latine (19 t.), Une époque des frères Margueritte, Les Derniers Scandales de Paris de Dubut de Laforest (37 t.); l'Histoire d'une société de René Béhaine (16 t.), Le Cycle de Lord Chelsea d'Abel Hermant, l'Histoire d'une famille sous la Troisième République de Robert Francis, Les Hauts-Ponts de Jacques de Lacretelle. 14. Voir A. Grésillon, La Mise en æuvre. Itinéraires génétiques, Paris, CNRS Éditions, 2008, p. 247 et $266-267$. 
effectué sur une pièce de théâtre. Dans les deux cas, l' "effet de réception », dont parle Daniel Ferrer dans Logiques du brouillon ${ }^{15}$, occupe une place importante. Car ce jeu ne peut se produire sans l'accord du lecteur ou du spectateur.

Comme dans le cas des ouvres théâtrales, la génétique des cycles romanesques doit se montrer capable de prendre des risques, si elle veut atteindre ses objectifs. Mais ces risques sont stimulants pour l'esprit. Ils témoignent de la fécondité de l'hypothèse génétique appliquée à l'étude des phénomènes littéraires.

Alain Pagès

15. Voir D. Ferrer, Logiques du brouillon. Modèles pour une critique génétique, Paris, Éditions du Seuil, 2011, p. 86.

Directeur des Cahiers naturalistes, Alain Pagès est responsable de «l'équipe Zola» de l'ITEM (CNRS-ENS). Il a publié récemment Zola et le groupe de Médan. Histoire d'un cercle littéraire (Perrin, 2014). Avec Brigitte Émile-Zola, il vient d'éditer, chez Gallimard, le recueil des lettres inédites de Zola à sa femme, Alexandrine, dont il a annoté les textes avec la collaboration de Céline Grenaud-Tostain, Sophie Guermès, Jean-Sébastien Macke et Jean-Michel Pottier.

pagesal2@orange.fr 